\title{
Effect of stellate ganglion block on laryngopharyngeal reflux disease
}

\author{
Ji Won Chung ${ }^{1}$, Hye Jung Chun ${ }^{1}$, Mi Soon Lee ${ }^{2}$, Ki Ryang Ahn ${ }^{1}$, Chun Sook Kim ${ }^{1}$, Kyu Sik Kang ${ }^{1}$, \\ Sie Hyeon Yoo ${ }^{1}$, Jin Hun Chung ${ }^{1}$, Nan-Seol Kim ${ }^{1}$, Yong Han Seo ${ }^{1}$, Hyung Youn Gong ${ }^{1}$, and \\ Yong Man Lee
}

Department of Anesthesiology and Pain Medicine, 'Soonchunhyang University Hospital,College of Medicine, Soonchunhyang University, Cheonan, ${ }^{2}$ Soonchunhyang University Hospital,College of Medicine, Soonchunhyang University, Bucheon, ${ }^{3}$ Department of Otorhinolaryngology, Soonchunhyang University Hospital, College of Medicine, Soonchunhyang University, Cheonan, Korea

Background: Laryngopharyngeal reflux (LPR) disease has many symptoms such as globus pharyngeus, excessive throat clearing and hoarseness. The aim of this study was to investigate the effect of stellate ganglion block (SGB) in addition to proton pump inhibitors (PPI) on LPR.

Methods: Fifty patients complaining of more than 3 typical LPR symptoms for over 3 months were enrolled in the study. The P group took PPI for 8 weeks. The SP group took PPI and interwent a series of 8 SGB procedure once a week during the period of treatment. The blocks were performed one at a time unilaterally on the right and left stellate ganglions by injecting $1 \%$ mepivacaine $6 \mathrm{ml}$. We evaluated the reflux symptom index (RSI) before treatment and following 4 weeks and 8 weeks of treatment in both groups.

Results: After 4 weeks of treatment, the RSI of the P group decreased, but not significantly, to $16.6 \pm 6.8$ compared with the baseline value of $19.2 \pm 2.7(\mathrm{P}=0.093)$, whereas the RSI of the SP group decreased significantly to $9.8 \pm$ 3.3 compared with the baseline value of $19.0 \pm 4.7(\mathrm{P}=0.000)$. After 8 weeks of treatment, the RSI of the $\mathrm{P}$ group decreased significantly to $13.7 \pm 6.7(\mathrm{P}=0.001)$ and the RSI of the SP group also decreased significantly to $7.7 \pm 3.4(\mathrm{P}=$ 0.000). There were significant differences in the RSI between the two groups after 4 weeks $(P=0.000)$ and 8 weeks $(P$ $=0.001)$ of treatment.

Conclusions: The symptoms of LPR improved earlier when PPI therapy was combined with SGB compared with PPI therapy alone. (Korean J Anesthesiol 2013; 64: 439-442)

Key Words: Laryngopharyngeal reflux, Proton pump inhibitors, Stellate ganglion block.

Received: May 7, 2012. Revised: 1st, July 15, 2012; 2nd, November 11, 2012. Accepted: November 28, 2012.

Corresponding author: Ji Won Chung, M.D., Department of Anesthesiology and Pain Medicine, Soonchunhyang University Hospital, College of Medicine, Soonchunhyang University, 23-20, Bongmyeong-dong, Cheonan 330-100, Korea. Tel: 82-41-570-2823, Fax: 82-41-573-3559, E-mail: wolftom@hanmail.net

(c) This is an open-access article distributed under the terms of the Creative Commons Attribution Non-Commercial License (http:// creativecommons.org/licenses/by-nc/3.0/), which permits unrestricted non-commercial use, distribution, and reproduction in any medium, provided the original work is properly cited. 


\section{Introduction}

Laryngopharyngeal reflux (LPR) is a condition that manifests upper aerodigestive tract symptoms due to the reflux of gastric contents to the laryngopharynx $[1,2]$. Globus pharyngeus (a sensation of a lump in the throat), heartburn, excessive throat clearing, cough, and hoarseness are the typical clinical manifestations $[1,2]$.

Recently, a report stated that $60 \%$ to $90 \%$ of LPR patients experienced symptomatic improvement after treatment with medications such as $\mathrm{H}_{2}$-blockers and proton pump inhibitors (PPI) [3]. These medications require long-term treatment, however, meaning that there is a limitation of early withdrawal of treatment if there is no improvement during the early stage of treatment.

The stellate ganglion block (SGB) is a maneuver applied in the treatment of various conditions, such as pain in the face and upper extremities, by internal medicine and ENT (Ears, nose and throat) departments.

Based on the hypothesis that more rapid and effective symptomatic improvement can be expected with SGB and PPI combination therapy compared with PPI monotherapy in LPR patients, the authors carried out a study to determine the effectiveness of SGB in the improvement of LPR symptoms using 2 study groups: a SGB and PPI combination therapy group and a PPI monotherapy group.

\section{Materials and Methods}

The study subjects were outpatients with LPR in the ENT department. Patients with the following conditions were excluded and treated with the consultation of the pain clinic: a medication history related to LPR symptoms, gastroesophageal reflux, rhinosinusitis, tonsillitis, lung disease, cervical disease, and esophageal disease.

A total of 50 patients were enrolled. Subjects manifested mild-to-moderate LPR symptoms according to the reflux symptom index (RSI) (Table 1) developed by Belafsky et al. [1]; they complained of more than 3 symptoms lasting more than 3 months and had RSI scores of 13-33 (16-25). The study subjects were divided into the $\mathrm{P}$ group, consisting of 25 patients receiving PPI monotherapy, and the SP group, consisting of 25 patients receiving SGB and PPI combination therapy. Due to withdrawal from the study, however, 22 patients in the $\mathrm{P}$ group and 20 patients in the SP group were included in the study. Informational documents, surveys, and consent forms were provided to the patients prior to the study in order to receive the approval of the hospital ethics committee. Then, with the informed consent of the patients, the study was initiated.

Information about the administration of SGB was provided to outpatients in the ENT department so that the patients could decide between PPI monotherapy and SGB and PPI combination therapy. The SP group received follow-up treatment at the pain clinic, and the $\mathrm{P}$ group received follow-up treatment at the outpatient clinic in the ENT department.

It was explained to the patients in the $\mathrm{P}$ group that the effect of PPI treatment takes more than 8 weeks and that side effects such as headache, nausea, diarrhea, stomachache, constipation, and vertigo could occur. Then the 8 -week treatment was initiated.

Lansoprazole 30 mg (Lanston ${ }^{\circledR}$, Jeilpharm, Seoul, Korea), the PPI agent, was administered once daily 30 minutes before breakfast for 8 weeks.

Patients in the SP group received an explanation about the method of the procedure, the degree of effects with various numbers of procedures, and side effects such as miosis, ptosis, upper extremities paralysis, dyspnea, and hematoma. After that, regional anesthesia was administered by an experienced pain clinic specialist while the patient was in the supine position with an extension of the cervical vertebra, so that the anterior tubercle of the cervical vertebra 6 (C6) transverse process could be easily palpated. Then the anterior tubercle of the C6 vertebra transverse process was palpated with a slightly opened mouth; the needle was inserted into the right angle and fixed, and

Table 1. Reflux Symptom Index

\begin{tabular}{|c|c|c|c|c|c|c|}
\hline \multicolumn{2}{|l|}{ Within the past month, how did the following problems affect you? } & \multicolumn{5}{|c|}{$0=$ No problem, $5=$ Severe problem } \\
\hline 1. Hoarseness or other voice problem & 0 & 1 & 2 & 3 & 4 & 5 \\
\hline 2. Clearing throat & 0 & 1 & 2 & 3 & 4 & 5 \\
\hline 3. Excess throat mucus or postnasal drip & 0 & 1 & 2 & 3 & 4 & 5 \\
\hline 4. Difficulty swallowing food, liquid, or pills & 0 & 1 & 2 & 3 & 4 & 5 \\
\hline 5. Coughing after eating or after lying down & 0 & 1 & 2 & 3 & 4 & 5 \\
\hline 6. Breathing difficulties or choking episodes & 0 & 1 & 2 & 3 & 4 & 5 \\
\hline 7. Troublesome or annoying cough & 0 & 1 & 2 & 3 & 4 & 5 \\
\hline 8. Sensation of something sticking in throat or lump in throat & 0 & 1 & 2 & 3 & 4 & 5 \\
\hline 9. Heartburn, chest pain, indigestion, or lump in throat & 0 & 1 & 2 & 3 & 4 & 5 \\
\hline \multicolumn{7}{|c|}{ Total } \\
\hline
\end{tabular}


the absence of any unusual sensation in blood aspiration and brachial was confirmed.

Treatment success was confirmed by the presence or absence of miosis and ptosis 5 minutes after the procedure. The patient was checked every week, alternating left and right eyes, and $1 \%$ mepivacaine $6 \mathrm{ml}$ was used. The RSI was documented by the patients in both groups prior to the treatment and at week 4 and week 8.

In the pilot study, the difference between groups was 6 , and the standard deviation was $5 ; \alpha=0.05$, and power $=0.8$. The sample size would be 10 patients with the clinically significant difference of $30 \%$, and considering the withdrawal rate of $50 \%$ in the SGB group, each group required 15 patients. To increase the $\mathrm{n}$ value, an additional 10 patients were recruited to make up 25 patients in each group.

All of the measured values were presented in mean \pm standard deviation, and SPSS (Version 14.0, SPSS Inc, USA) was used for statistical analysis. The RSI group difference was assessed with an independent t-test, and pre- and post-treatment group differences were assessed with a paired t-test. Results were considered to be statistically significant when the $P$ value was less than 0.05 . The post hoc test and normality assumption were conducted with a histogram. The Q-Q plot/KolmogorovSmirnov test, Shapiro-Wilk test, and equal variance test were conducted with Levene's test.

\section{Results}

Forty-two out of 50 patients were recruited as study subjects, and 3 out of 25 patients in the SP group discontinued the treatment as they did not opt for SGB, even with the detailed explanation of

Table 2. Demographic Data

\begin{tabular}{lcc}
\hline & P group $(\mathrm{n}=22)$ & SP group $(\mathrm{n}=20)$ \\
\hline Sex $(\mathrm{M} / \mathrm{F})$ & $7 / 15$ & $11 / 9$ \\
Age $(\mathrm{yr})$ & $47.8 \pm 14.2$ & $49.9 \pm 15.2$ \\
Weight $(\mathrm{kg})$ & $58.4 \pm 5.8$ & $56.7 \pm 3.9$ \\
Height $(\mathrm{cm})$ & $163.2 \pm 8.2$ & $162.8 \pm 8.0$ \\
\hline
\end{tabular}

Values are expressed as mean \pm SD except sex. n: number of patients, P group: proton pump inhibitor, SP group: stellate ganglion block + proton pump inhibitor. the prevalence of Horner's syndrome, which accompanies side effects such as miosis, ptosis and enophthalmos after the SGB procedure. Two patients dropped out of the study due to the loss of follow-up. None of the 25 patients in the P group had to stop the treatment due to medication side effects, but 3 patients dropped out of the study due to the loss of follow-up.

No significant differences between the groups were found in sex, age, weight, or height (Table 2). The RSI prior to the treatment was $19.2 \pm 2.7$ in the P group and $19.0 \pm 4.7$ in the SP group, with no between-group difference. At week 4 , however, the RSI was $16.6 \pm 6.8$ in the P group and $9.8 \pm 3.3$ in the SP group, with the SP group showing a significant reduction. At week 8 , a reduction was seen in both groups, to $13.7 \pm 6.7$ in the P group and $7.7 \pm 3.4$ in the SP group (Table 3 ). In comparing the RSI reductions between the 2 groups to compare the treatment efficacy, a significant difference was found at week 4 and week 8 .

\section{Discussion}

The prevalence of LPR is known to be quite high, affecting up to $10 \%$ to $20 \%$ of outpatients in the ENT department $[4,5]$, but the number of patients at the pain clinic is low.

The best diagnostic tool for LPR is ambulatory 24-hour double-probe $\mathrm{PH}$ monitoring, but diagnosis takes a long time, the cost is high, there is a high chance of false positive, and the measured $\mathrm{pH}$ outcome and the degree of symptoms often do not match due to individual differences in the sensitivity of the esophagus and laryngopharynx [5]. Therefore, the RSI, which converts LPR symptoms and manifestations into scores, and the reflux finding score (RFS), the score for laryngopharynx reflux manifestation, are used as diagnostic tools [1].

The RFS with a laryngendoscope is hard to carry out in the outpatient clinic, but the RSI developed by Belafsky et al. [1] is known to reflect improvement in symptoms even though it is a survey done by patients, which can be a subjective measure. An RSI score greater than 13 is considered abnormal $[6,7]$.

Other treatment options for LPR patients are as follows: patient education, lifestyle changes, antacids, $\mathrm{H} 2$ receptor antagonists, gastrointestinal stimulants, PPI, and antireflux surgery [8].

In acid production from gastric cells, the last step for the production of hydrogen ions is the hydrogen ion pump, where

Table 3. Change of Reflux Symptom Index

\begin{tabular}{lcccc}
\hline & $\begin{array}{c}\text { Before } \\
\text { treatment }\end{array}$ & $\begin{array}{c}\text { 4 weeks } \\
\text { after treatment }\end{array}$ & $\begin{array}{c}\text { 8 weeks } \\
\text { after treatment }\end{array}$ & $\begin{array}{c}\text { P value } \\
(8 \text { weeks after }) \\
(4 \text { weeks after) }\end{array}$ \\
\hline P group & $19.2 \pm 2.7$ & $16.6 \pm 6.8$ & $13.7 \pm 6.7$ & 0.093 \\
SP group & $19.0 \pm 4.7$ & $9.8 \pm 3.3$ & $7.7 \pm 3.4$ & 0.000 \\
P value & $0.815^{*}$ & $0.000^{\dagger}$ & $0.001^{\dagger}$ & 0.000 \\
\hline
\end{tabular}

Values are mean \pm SD. P group: proton pump inhibitor group, SP group: stellate gangoion block + proton pump inhibitor group. ${ }^{*} \mathrm{P}$ value $>0.05$ compared with before treatment. ${ }^{\dagger} \mathrm{P}$ value $<0.05$ between groups. 
the conversion of potassium and hydrogen takes place; this step is carried out by $\mathrm{H}^{+} \mathrm{K}^{+}$ATPase. PPI reduces the production of acid in this last step and exerts a reduction effect in gastric mucosity.

Apart from lansoprazole, other PPIs are available, such as omeprazole and pantoprazole. As they are not stable in acid, they are administered as agonists.

Unlike prokinetic agents, PPI can be used as treatment to address the cause of the condition, as PPI completely blocks the last step of the gastric acid secretion that causes LPR [9]. In addition, there is no development of tolerance, as is normally seen in $\mathrm{H} 2$ receptor antagonists [10].

Wo et al. [5] and DelGaudio and Waring [11] report that only hoarseness symptoms improve with 4 weeks of PPI treatment, and symptoms such as throat clearing and cough take 8 weeks of PPI administration to see improvement in symptoms. Patients with no symptomatic improvement may require antireflux surgery.

The authors carried out this study by administering PPI longer than 8 weeks and SGB more than 8 times. As a result, at week 4 there was no significant reduction of RSI in the P group, with a reduction of 2.6, while a significant reduction of 9.2 was observed in the SP group at week 4. In addition, at week 8, significant reductions of 5.5 and 11.3 were observed in groups $\mathrm{P}$ and SP, respectively; based on these results, the combination of SGB and PPI treatment may lead to early symptomatic improvement (Table 3).

SGB is a maneuver applied to various conditions, and Kageshima et al. [12] reported improvement in patients with recurrent tonsillitis even with repeated surgeries and medication administration. The authors initiated the study with the hypothesis that SGB would improve LPR.

The most effective treatment cycle of SGB has not been clearly established; the authors took into consideration the difficulty of visiting the outpatient clinic, and the cycle was planned to be once weekly and greater than 8 cycles.

SGB increases the amount of blood flow without major fluctuations in blood pressure, heart rate, and cardiac output; it blocks the pain related to the visceral afferent pathway by stabilizing the sympathetic hypertonic state, which exerts effects on the endocrine system and immune system [13].

As seen in the above patients, SGB resulted in relaxation of the laryngopharynx, decreased gastric acid secretion, and circulation improvement in surrounding tissues due to the increase in blood flow and stabilization of the sympathetic nervous system. The authors conclude that these effects led to the improvement in discomfort and pain in the laryngopharynx.
In conclusion, SGB resulted in improvement of LPR symptoms in the early stage in mild-to-moderate patients, although the authors could not identify the clear mechanisms of action of SGB for the improvement of LPR symptoms.

However, a greater sample size with patients suffering from severe symptoms needs to be studied to reach a firmer conclusion about the effect of combination treatment with SGB and PPI on LPR. A further prospective study is warranted to determine differences in gastric acid reduction from vagus nerve block, which is a derived effect of SGB, as well as to explore psychological factors, the relationship between other medications and the number of treatments, and the effect of treatment duration.

\section{References}

1. Belafsky PC, Postma GN, Amin MR, Koufman JA. Symptoms and findings of laryngopharyngeal reflux. Ear Nose Throat J 2002; 81 (9 Suppl 2): 10-3.

2. Noordzij JP, Khidr A, Desper E, Meek RB, Reibel JF, Levine PA. Correlation of $\mathrm{pH}$ probe-measured laryngopharyngeal reflux with symptoms and signs of reflux laryngitis. Laryngoscope 2002; 112: 2192-5.

3. Metz DC, Childs ML, Ruiz C, Weinstein GS. Pilot study of the oral omeprazol test for reflux laryngitis. Otolaryngol Head Neck Surg 1997; 116: 41-6.

4. Ford CN. Evaluation and management of laryngopharyngeal reflux. JAMA 2005; 294: 1534-40.

5. Wo JM, Grist WJ, Gussack G, Delgaudio JM, Waring JP. Empiric trial of high-dose omeprazole in patients with posterior laryngitis: a prospective study. Am J Gastroenterol 1997; 92: 2160-5.

6. Belafsky PC, Postma GN, Koufman JA. Validity and reliability of the reflux symptom index (RSI). J Voice 2002; 16: 274-7.

7. Belafsky PC, Postma GN, Koufman JA. The validity and reliability of the reflux finding score (RFS). Laryngoscope 2001; 111: 1313-7.

8. Choi HS. Laryngopharyngeal reflux disease. The 2nd digestive disease research lecture. Korea University Digestive Research Institute 1999: 25-32.

9. Shaw GY, Searl JP. Laryngeal manifestations of gastroesophgeal reflux before and after treatment with omeprazole. South Med J 1997; 90: 1115-22.

10. Wilder-Smith CH, Ernst T, Gennoni M, Zeyen B, Halter F, Merki HS. Tolerance to oral H2-receptor antagonist. Dig Dis Sci 1990; 35: 97683.

11. Delgaudio JM, Waring JP. Empiric esomeprazole in the treatment of laryngopharyngeal reflux. Laryngoscope 2003; 113: 598-601.

12. Kageshima K, Wakasugi B, Ooseto K, Yuda Y, Shiotani M. The effect of stellate ganglion block therapy on tonsillitis. Masui 1992; 41: 2002-4.

13. The Korean Pain Society. Pain medicine. 3rd ed. Seoul, Koonja publishing Inc. 2007, pp 583-7. 